\title{
Widefield and total internal reflection fluorescent structured illumination microscopy with scanning galvo mirrors
}

\author{
Youhua Chen \\ Ruizhi Cao \\ Wenjie Liu \\ Dazhao Zhu \\ Zhiming Zhang \\ Cuifang Kuang \\ $\mathrm{Xu}$ Liu
}




\title{
Widefield and total internal reflection fluorescent structured illumination microscopy with scanning galvo mirrors
}

\author{
Youhua Chen, ${ }^{\mathrm{a}, \mathrm{b}}$ Ruizhi Cao, ${ }^{\mathrm{a}}$ Wenjie Liu, ${ }^{\mathrm{a}}$ Dazhao Zhu, ${ }^{\mathrm{a}}$ Zhiming Zhang, ${ }^{\mathrm{a}}$ Cuifang Kuang, ${ }^{\mathrm{a}, \mathrm{c}, \star}$ and Xu Liu ${ }^{\mathrm{a}, \mathrm{c}, *}$ \\ aZhejiang University, State Key Laboratory of Modern Optical Instrumentation, College of Optical Science and Engineering, Hangzhou, China \\ ${ }^{b}$ North University of China, Key Laboratory of Instrumentation Science and Dynamic Measurement of Ministry of Education, Taiyuan, China \\ cShanxi University, Collaborative Innovation Center of Extreme Optics, Taiyuan, China
}

\begin{abstract}
We present an alternative approach to realize structured illumination microscopy (SIM), which is capable for live cell imaging. The prototype utilizes two sets of scanning galvo mirrors, a polarization converter and a piezo-platform to generate a fast shifted, $s$-polarization interfered and periodic variable illumination patterns. By changing the angle of the scanning galvanometer, we can change the position of the spots at the pupil plane of the objective lens arbitrarily, making it easy to switch between widefield and total internal reflection fluorescent-SIM mode and adapting the penetration depth in the sample. Also, a twofold resolution improvement is achieved in our experiments. The prototype offers more flexibility of pattern period and illumination orientation changing than previous systems. ๑ 2018 Society of Photo-Optical Instrumentation Engineers (SPIE) [DOI: 10.1117/1.JBO.23.4.046007]
\end{abstract}

Keywords: fluorescence microscopy; total internal reflection; structure illumination; super-resolution; scanning galvo mirrors.

Paper 180030RR received Jan. 17, 2018; accepted for publication Apr. 3, 2018; published online Apr. 24, 2018.

\section{Introduction}

Fluorescence microscopy has a significant impact on cell biology by imaging various cells with visible light, which is noninvasive to biological samples. The classical Abbe diffraction limit in fluorescence microscopy (roughly $200 \mathrm{~nm}$ ) has been a barrier for quite a long time. Remarkable advances have been made in recent decades in science and technology to break the diffraction limit and obtain the nanoscale resolution. The super-resolution (SR) techniques ${ }^{1}$ include stimulated emission depletion microscopy, ${ }^{2}$ structured illumination microscopy (SIM) ${ }^{3-5}$ single molecule localization-based microscopy, ${ }^{6-8}$ and so forth, paving the way for significant progress in biological research.

Among them, SIM doubles the resolution of conventional microscopes by exciting fluorescence with spatially patterned light and collects the down-modulated frequency information that is not accessible in conventional microscopes. It is superior to other SR techniques in terms of speed and suitability for live samples and is a widefield nonscanning technique, requiring only several images to reconstruct a high-resolution image, without high and harmful illumination intensities. ${ }^{9}$ As the SIM concept was proposed, a couple of system types have been established, ${ }^{10-16}$ and some notable achievements like threedimensional (3-D) SR live cell imaging have been made in the biological application.

For all the interference-based SIM systems, it is necessary to produce two (two-dimensional SIM)/three beams (3-D SIM) that are focused on the rear focal plane of the objective lens, which can then be collimated through the objective lens and produce interference fringes at the intersection. To achieve this, typical commercial SIM systems (Nikon N-SIM \& Zessi ElyraSIM) using a transmission phase grating produce the sinusoidal

*Address all correspondence to: Cuifang Kuang, E-mail: cfkuang @ zju.edu.cn; Xu Liu, E-mail: liuxu@zju.edu.cn pattern, translating by a piezoelectric actuator, and rotating by a mechanical stage. ${ }^{10}$ Obviously, the mechanical movement of the grating requires precise servo system cooperation and limits the acquisition speed to several seconds.

To solve this problem, a phase-only spatial light modulator $(\mathrm{SLM})^{11}$ is employed to produce and translate the one-dimensional (1-D) interference patterns instead of the physical grating. This scheme has the advantages of allowing one to tune the illumination parameters over a broad range, eliminating the mechanical movement, and so on. But due to the limited refreshing speed of SLM (40 to $60 \mathrm{~Hz}$, typically), the frame rate of SIM is still well below the requirements of live cell imaging. Benefiting from the development of SLM, the ferroelectric liquid crystal on silicon (FLCOS) with a high refreshing rate $(\sim 1 \mathrm{kHz})$ was invented and applied. Then with a carefully designed polarization rotator to keep the $s$-polarization that is the key point to obtain high-contrast fringes, SIM finally achieves a video frame rate and enables one to image live biological samples with high quality. ${ }^{12-16}$

But, high-power lasers are needed for the FLCOS-based SIM because of the low diffraction efficiency of FLCOS $(<10 \%$, typically), and due to the finite and discrete duty cycles of the grating loaded on the SLM, the choice of the pattern has to satisfy some constraints. Usually, such optical scheme requires a relatively complex pattern setting of the grating (such as shearing the grating to change the grating vector, combine the vertically interleaving and horizontal patterns together, and so forth ${ }^{11,14,15}$ ) on the SLM to achieve near-identical line spacings at different illumination orientations and equispaced phase shift, which is particularly important in total internal reflection fluorescence (TIRF) SIM. In many cases, observing and comparing the sample at different TIR angle plays an important role in biological research because the different penetration depths will bring

$1083-3668 / 2018 / \$ 25.00$ @ 2018 SPIE 
different features of the tissues, ${ }^{17}$ and SLM-based SIM is not very convenient to change the illumination angle continuously to satisfy this requirement.

Furthermore, the modulation depth of the patterns plays an important role in SIM, and the $s$-polarized incident lights at the rear focus plane are preferred. ${ }^{18}$ Thus, the digital micromirror device based SIM $^{19}$ that utilizes the incoherent pattern is not suitable for high-NA objective lens, and the off-axis-Michelsoninterferometer-type SIM in Ref. 13 using circular polarization to produce the patterns sacrifices the modulation depth and the quality of the SR image, in spite of overcoming the disadvantages of SLM by a piezo-driven tip-tilt mirror.

In this article, we provide an alternative approach to realize high modulation depth patterns ( $s$-polarization interference) SIM system using galvo mirrors, piezo-platform, and polarization converter. It can arbitrarily increase the angle of incidence of the illumination light up to the TIR and shifting the phase of pattern at a very high speed (depends on the speed of response of the galvo mirror), making it easier to switch between widefield $\&$ TIRF-SIM, offering more convenience in adapting the penetration depth of the biological tissues.

\section{Optical Setup}

As shown in Fig. 1(a), the SIM imaging system was built based on a Nikon Ti-series microscope. The polarized excitation laser beam (shown as green) was coupled into the polarization maintaining single-mode fiber (Oz Optics, core size $3 \mu \mathrm{m}$, NA 0.11 ) and collimated by the beam collimator (CL, Thorlabs, ZC618FC). The incident beam can be separated into two beams with the same intensity by the polarizing beam splitter
(PBS, Thorlabs, CCM1-PBS251/M) via rotating the half-wave plate (HWP1, Thorlabs, AQWP05M-600). Though the mirrors (M1, M2, M3, and M4), the beams are reflected into the corresponding scanning unit. The M2 is placed on the 1-D manual adjustable table [not shown in Fig. 1(a)] to adjust the optical path difference between the two-path beams to keep them in the coherence length.

There are two groups of completely symmetric scanning units in the system - the orange area is shown in Fig. 1(a). Each scanning unit consists of two scanning galvo mirrors (SMX, SMY, Cambridge Technology, CTI 8310k) and two spherical mirrors (SM1 and SM2). The focal lengths of SM1 and SM2 are 100 and $50 \mathrm{~mm}$, respectively. The galvo mirrors and spherical reflectors form a 4f system, in which SM1 and SM2 are used to project the illumination spot to the position of the rotation axes of the SMY and keep the spot fixed (fixed point) at the center of SMY while the SMX rotates. The chief ray of each beam is parallel to the optical axis again through the scanning lens (SL, Thorlabs, SL50-CLS2). The half-wave plate (HWP2, Thorlabs, AQWP05M-600) before the scanning unit 2 is applied to keep the polarization direction of the two beams consistent. The 4f scanning unit setup using two spherical mirrors (SM1 and SM2) can avoid the problem of noncoplanar line optical path in a refraction-type $4 \mathrm{f}$ scanning system, and meanwhile can keep its advantages, like keeping the fixed point at the last scanning mirror and flat and wide scanning range, and so on.

The polarization of the illumination beams plays an important role in the generation of a fine interference pattern, especially in the TIRF-SIM system whose modulation frequency is sufficiently high. Hence, we introduce the polarization

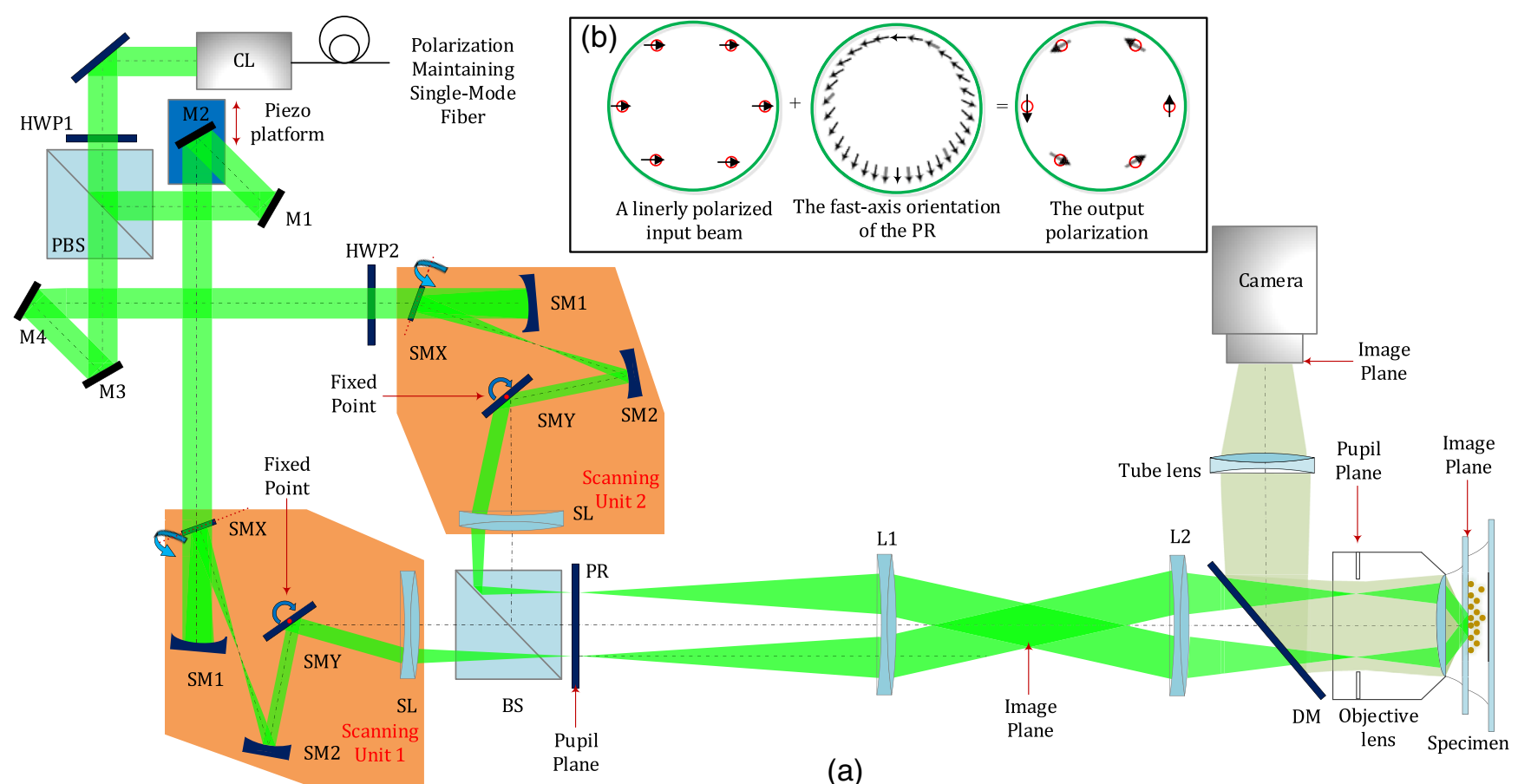

Fig. 1 (a) Optical scheme of the system. (b) The schematic diagram of how the polarization converter (PR) rotates the polarization of linearly polarized input beam into $s$-polarization at the pupil plane of the objective lens. The dotted dashed line is the optical axis of the system. CL, beam collimator; HWP1, HWP2, half-wave plate; PBS, polarizing beam splitter cube; BS: beam splitter cube; M1, M2, M3, M4, mirrors; SM1, SM2, spherical mirrors; SMX, SMY, scanning galvo mirror; SL, scanning lens; PR, polarization converter; L1, L2, lens; and DM, dichroic mirror. The red circles in the subfigure denote the focuses of the incident beams introduced by the BS. The arrows represent the polarization of the beams. The arrow in the middle of Fig. 1(b) shows the fast axis orientation of the polarization converter. 

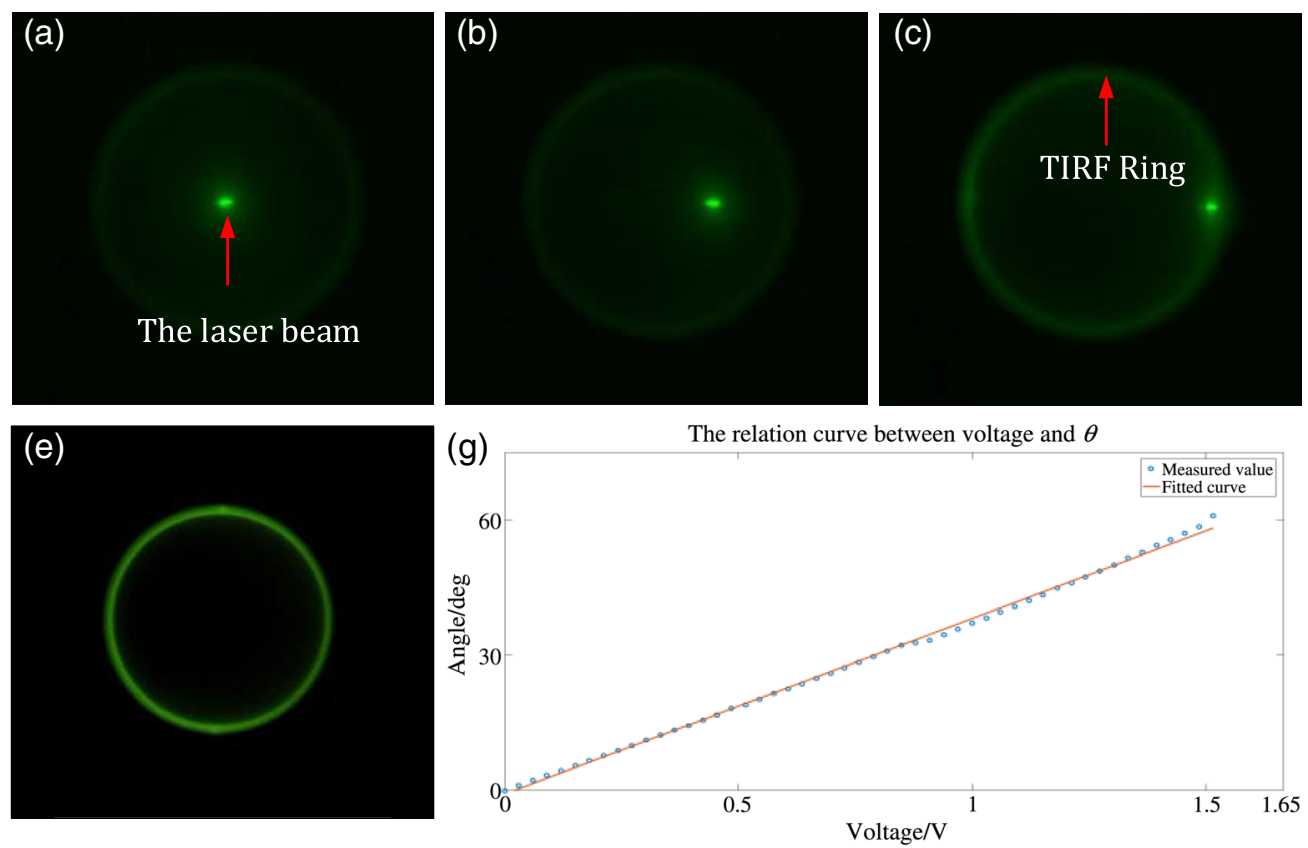

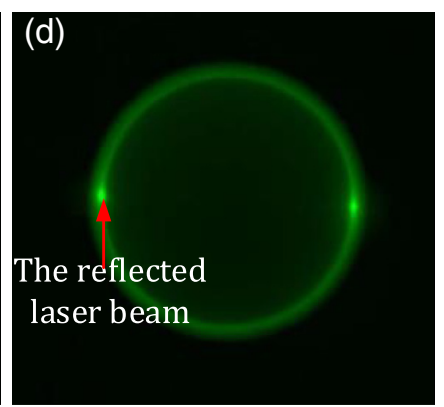

Fig. 2 The experimental images of the BFP of the objective and the calibrating curve for the voltage and $\theta$. (a) $\theta=0$ deg, $\alpha=0$ deg, voltage $=0 \mathrm{~V}$; (b) $\theta=48 \mathrm{deg}, \alpha=0 \mathrm{deg}$, voltage $=1.2 \mathrm{~V}$; (c) $\theta=60$ deg, $\alpha=0 \mathrm{deg}, \quad$ voltage $=1.50 \mathrm{~V} ;$ (d) $\theta=62 \mathrm{deg}, \quad \alpha=0 \mathrm{deg}$, voltage $=1.55 \mathrm{~V} ;$ (e) $\theta=62$ deg, $\alpha=90 \mathrm{deg}$, voltage $=1.55 \mathrm{~V}$; (f) $\theta=62 \mathrm{deg}, \alpha=60 \mathrm{deg}$, voltage $=1.55 \mathrm{~V}$; and (g) the relation curve between voltage and $\theta$.

converter (PR, Thorlabs, WPV10L) at the conjugated pupil plane right behind the beam splitter (BS, Thorlabs, CCM1BS013) to generate the $s$-polarized beam on the sample plane. Figure 1(b) shows the basic principle of how the PR rotates the polarization of linearly polarized input beam into $s$-polarization at the pupil plane. The small red circle represents the cross section of the beam and the large green circle represents the entrance pupil of the PR, which size is the same as the outer size of the PR. From left to the right, the black arrow represents the polarization direction of the incident beam, the fast axis of the PR, and the polarization direction of the outgoing beam. Through adapting the center of PR to coincide with the optical axis of the system and rotating the fast axis of PR to a proper direction, all parts of each focused beam will be simultaneously converted to $s$-polarization.

The note is that the PR is a vortex phase plate when the incident light is parallel and is distributed symmetrically around the center of PR. But, if the incident beam is focused as a point on the PR, then, all the polarization of the beam will be converted to the corresponding direction depending on the focused position and the fast axis of the PR, by adapting the center of the PR to coincide with the optical axis of the system and rotating the fast axis to a proper direction, the $s$-polarization will be achieved very easily without any time lapse than the PR combined with liquid crystal and one-fourth wave plate and much cheaper than the PR combined with Pockels cell.

After PR, the desired two beams are refocused to the centrosymmetric points near the edges of the pupil plane of the microscope objective $(100 \times / 1.49$ TIRF, Nikon) by two-tube lenses (L1, L2, Thorlabs, TTL200-A, $f=200 \mathrm{~mm}$ ). Each beam is recollimated by the objective lens and hit the cover slip surface at a tilt angle $\theta$. Through the tip/tilt of SMX and SMY in each scan unit, $\theta$ will be changed continuously. When $\theta$ is less than the critical angle, the widefield-SIM mode works, else the evanescent waves from the two beams extend $\sim 100 \mathrm{~nm}$ into the specimen and interfere to make the TIRF-SIM mode work. The phase shift of the illumination pattern is realized by M2, which is fixed on a 1-D piezo-platform (PI, P-753). The emission light (shown as yellow) from the specimen is collected by the electron multiplying charge coupled device (EMCCD, Andor, iXon Ultra 888) after the dichroic mirror (DM, Chroma, C174298) as in a conventional fluorescence microscope.

The galvo mirrors and the piezo-platform are chosen due to their high-speed $\mathrm{kHz}$ rate angle tuning capability, which made our system have the capacity to change the orientation and phase of the illumination pattern very quickly, and meanwhile have the potential to achieve SR imaging at very high frame rate. The modulation depth of the pattern is controlled by adjusting the polarization direction of the incident beam existing from the scanning units and the fast axis of PR, and let them fit in the relationship of Fig. 1(b). Unlike the SIM system mentioned above, this setup can realize $s$-polarization at arbitrary orientation without mechanical movement that will be beneficial for future use, like further improving the spectrum integrity by illuminating in five or more orientations and applying pattern-illuminated Fourier ptychography algorithm's in SIM and so on.

For the aligning and calibrating the instrument, there are already some related literatures that describe similar works. ${ }^{20,21}$ Here, we chose to record a series of images of the BFP of the objective at different azimuthal angles $\alpha$ to finish the alignment and calibration, while the position of laser spot in the image series is the function of $\theta$ according to the Descartes' laws. To achieve this, we first removed the eyepiece of Nikon Ti1 and replaced it with a C-mount camera combined with a convex lens, then placed the sample of a monolayer of fluorescent particles (F8830) fixed on the glass/water interface. The experimental results and the calibrating curve for the voltage and $\theta$ are shown in Fig. 2, from Figs. 2(a)-2(d), the $\theta$ was increasing 
linearly with the voltage, achieved the critical angle at Fig. 2(c) and the TIRF angle at Fig. 2(d).

The note is that we can observe the appearance of reflected light spots when TIRF occurs, which is more accurate for TIRF angle calibrating than measuring the spots position on the laboratory roof. Furthermore, for our TIRF-SIM system, the initial alignment is that we only need to adjust the two lights to exit from the center of the BFP, as the status shown in Fig. 2(a), then the symmetry can be guaranteed by the galvanometer with proper voltage, and the interference will happen sequentially. In addition, the asymmetry of the system for different azimuthal angles also can be compensated by adjusting the voltages of the galvanometers.

\section{Reconstitution Algorithm and Procedure}

Assuming that the three-step phase shifting strategy is applied, the image processing of SIM can be expressed by the following equation:

$$
\begin{aligned}
{\left[\begin{array}{c}
\tilde{D}_{1}(\mathbf{k}) \\
\tilde{D}_{2}(\mathbf{k}) \\
\tilde{D}_{3}(\mathbf{k})
\end{array}\right]=} & {\left[\begin{array}{ccc}
1 & \frac{m}{2} e^{i \varphi_{1}} & \frac{m}{2} e^{-i \varphi_{1}} \\
1 & \frac{m}{2} e^{i \varphi_{2}} & \frac{m}{2} e^{-i \varphi_{2}} \\
1 & \frac{m}{2} e^{i \varphi_{3}} & \frac{m}{2} e^{-i \varphi_{3}}
\end{array}\right]\left[\begin{array}{c}
\tilde{S}(\mathbf{k}) \tilde{H}(\mathbf{k}) \\
\tilde{S}\left(\mathbf{k}-\mathbf{k}_{0}\right) \tilde{H}(\mathbf{k}) \\
\tilde{S}\left(\mathbf{k}+\mathbf{k}_{0}\right) \tilde{H}(\mathbf{k})
\end{array}\right] } \\
= & {\left[\begin{array}{ccc}
1 & \frac{m}{2} e^{i \varphi_{1}} & \frac{m}{2} e^{-i \varphi_{1}} \\
1 & \frac{m}{2} e^{i \varphi_{2}} & \frac{m}{2} e^{-i \varphi_{2}} \\
1 & \frac{m}{2} e^{i \varphi_{3}} & \frac{m}{2} e^{-i \varphi_{3}}
\end{array}\right]\left[\begin{array}{c}
\tilde{C}_{0}(\mathbf{k}) \\
\tilde{C}_{+}(\mathbf{k}) \\
\tilde{C}_{-}(\mathbf{k})
\end{array}\right], }
\end{aligned}
$$

where $\sim$ denotes the corresponding Fourier transform, $D_{i}$ denotes the data of $i$ 'th caption, $m$ is the modulation depth, $\varphi_{i}$ is the phase of the $i$ 'th pattern, $\mathbf{k}_{0}$ is the modulation wavevector, $S$ is the fluorescence distribution, and $H$ is the point spread function, respectively.

To obtain a high-fidelity image with enhanced resolution, the phases and the modulation wavevector should be determined first. As for TIRF-SIM, the modulation frequency is usually close to or larger than the cutoff frequency of the detection optical transfer function (OTF). Hence, the peak searching scheme for the parameters' estimation is not suitable here. We apply the cross-correlation method for the estimation of the phase and the wavevector. As demonstrated in Ref. 22, we can estimate the modulation wavevector from the following correlation result:

$$
\begin{gathered}
\tilde{C}_{0} \otimes \tilde{C}_{+}\left(\mathbf{k}^{\prime}\right)=\int_{F} \operatorname{conj}[\tilde{S}(\mathbf{k}) \tilde{H}(\mathbf{k})] \\
\cdot \tilde{S}\left(\mathbf{k}-\mathbf{k}_{0}+\mathbf{k}^{\prime}\right) \tilde{H}\left(\mathbf{k}+\mathbf{k}^{\prime}\right) \mathrm{d} \mathbf{k}
\end{gathered}
$$

where $\otimes$ denotes the cross-correlation operation and $F$ stands for the Fourier domain. A local maximum will appear when $\mathbf{k}^{\prime}=\mathbf{k}_{0}$ in Eq. (2) and hence the modulation wavevector can be estimated. Here, we assume the separation between every two phases is $\frac{2}{3} \pi$, thus the relation between the separated components $R$ and $C$ is given by

$$
\left[\begin{array}{c}
\tilde{R}_{0}(\mathbf{k}) \\
\tilde{R}_{+}(\mathbf{k}) \\
\tilde{R}_{-}(\mathbf{k})
\end{array}\right]=\left[\begin{array}{ccc}
1 & \frac{m}{2} e^{0} & \frac{m}{2} e^{0} \\
1 & \frac{m}{2} e^{i \frac{i}{3} \pi} & \frac{m}{2} e^{-i \frac{2}{3} \pi} \\
1 & \frac{m}{2} e^{-i \frac{2}{3} \pi} & \frac{m}{2} e^{i \frac{2}{3} \pi}
\end{array}\right]^{-1}\left[\begin{array}{ccc}
1 & \frac{m}{2} e^{i \varphi_{0}} & \frac{m}{2} e^{-i \varphi_{0}} \\
1 & \frac{m}{2} e^{i\left(\frac{2}{3} \pi+\varphi_{0}\right)} & \frac{m}{2} e^{-i\left(\frac{2}{3} \pi+\varphi_{0}\right)} \\
1 & \frac{m}{2} e^{i\left(-\frac{2}{3} \pi+\varphi_{0}\right)} & \frac{m}{2} e^{-i\left(-\frac{2}{3} \pi+\varphi_{0}\right)}
\end{array}\right]\left[\begin{array}{c}
\tilde{C}_{0}(\mathbf{k}) \\
\tilde{C}_{+}(\mathbf{k}) \\
\tilde{C}_{-}(\mathbf{k})
\end{array}\right]=\left[\begin{array}{c}
\tilde{C}_{0}(\mathbf{k}) \\
e^{i \varphi_{0}} \tilde{C}_{+}(\mathbf{k}) \\
e^{-i \varphi_{0}} \tilde{C}_{-}(\mathbf{k})
\end{array}\right] .
$$

Thus, the initial phase can be also estimated via the correlation result. We can reconstruct the SIM result according to the parameters estimated above.

Figure 3 shows a typical procedure of acquiring and processing in SIM. Once a set of the modulation parameters (such as the modulation frequency and pattern orientations) is chosen, the electrical field at the Fourier plane is determined. Different voltages will thus be applied onto the galvo to generate the desired illumination pattern. The reconstruction parameters of a set of images are estimated via cross correlation of the predemodulated components, as described in Eq. (3). The different frequency bands of the sample are then shifted to their correct position, through which the frequency support of the reconstructed image is extended.

\section{Experimental Results}

To demonstrate the feasibility of the proposed system, we have conducted the following experiments, as shown in Figs. 4-6.

First, we imaged the 100-nm yellow-green (excitation wavelength: $505 \mathrm{~nm} / \mathrm{emission}$ wavelength: $515 \mathrm{~nm}$ ) fluorescent beads (F8803, Life Technologies) under TIRF-SIM mode, with an EMCCD exposure time of $50 \mathrm{~ms}$ per image. The results in Fig. 4 show that the reconstructed image by our system yields clearly distinct beads. This can be found quantitatively from the cross-section plots, given for four close-by beads (indistinguishable in widefield) in Fig. 4(c). The full-width at half-maximum (FWHM) of a single 100-nm diameter fluorescent bead decreases from 230 to $117 \mathrm{~nm}$, yielding an approximately twofold resolution improvement. This agrees well with the theoretical enhancement factor, estimated based on the TIR wave vector and the numerical aperture of the detection objective (NA = 1.49). ${ }^{16,23}$ The tissue (Abberior STAR 635P, excitation wavelength: $634 \mathrm{~nm} /$ emission wavelength: $651 \mathrm{~nm}$ ) results are shown in Fig. 5. The proposed system produces a striking resolution improvement over deconvolved conventional TIRF image. The FWHM of isolated microtubules reaches $105 \mathrm{~nm}$ in the TIRF-SIM reconstruction, and Fig. 5(c) shows a typical cross-section profile of the data.

Then, we demonstrated the capability of our system of changing the illumination angle $\theta$ as well as the pattern orientation and thus making the system work at widefield/TIRF-SIM mode continuously and conveniently. From Figs. 6(a)-6(c), the $\theta$ gradually increases and exceeds the critical angle $(\theta=60 \mathrm{deg})$ in Fig. 6(d). The corresponding FWHMs of the PSFs are shown in Fig. 6(f), which agrees well with the theoretical enhancement of SIM. When the $\theta$ is small, the frequency components are just a little bit above the system's OTF (dashed yellow circle in Fig. 6), and as $\theta$ increases, the frequency components also gradually increase. Note that the illumination conditions of Figs. 6(b) and 6(c) are typical widefield and TIRF-SIM, respectively. 


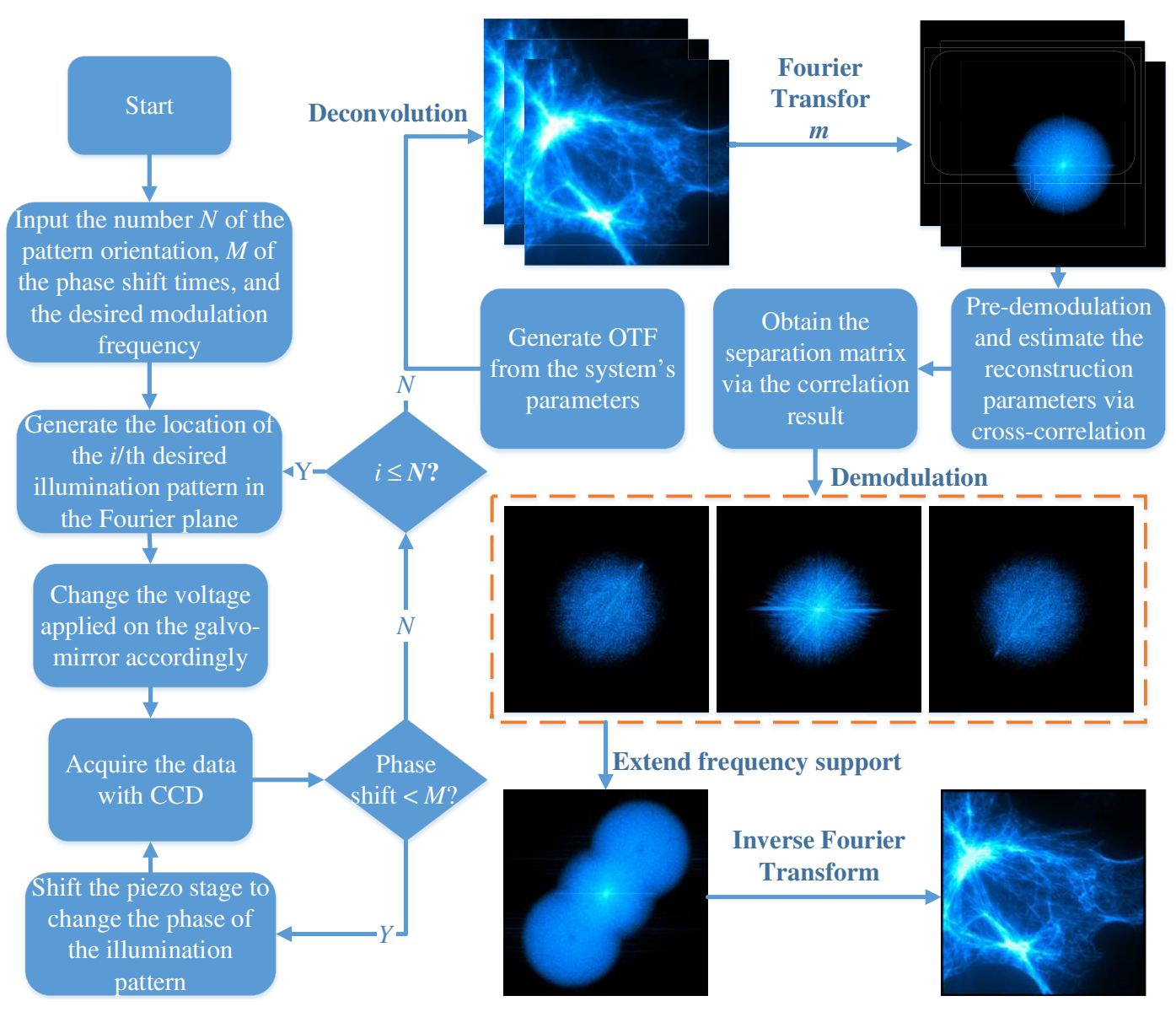

Fig. 3 The procedure to acquire and process the data of SIM. Three images that share the same modulation frequency and pattern orientations are assumed to be the data in this case. The parameters such as the modulation frequency, modulation depth, and initial phase can be estimated from the correlation result. And hence one can obtain the inverse matrix to demodulate these components properly.

Figures 6(c)-6(e) show the capability of the multiorientation pattern illumination using our system at the same $\theta=62 \mathrm{deg}$, and the orientations are 3,5 , and 20 with equiangular interval, respectively. Figures $6(\mathrm{~g})$ and $6(\mathrm{~h})$ show the reconstructed results of beads under the conditions shown in Figs. 6(c) and 6(e), respectively. Comparing these results, we can see that the more complete the spectrum is, the more rounded the particles are. Therefore, adding more illumination orientation appropriately will reduce the artifacts in SIM reconstruction, although more illuminating directions increase the raw data number and decrease the SR imaging speed, it can improve the reconstructed SR image quality and bring less artifacts, which is very important when doing the biological tissue analysis. $^{24,25}$

The system also has some optical sectioning capacity, as shown in Fig. 7. The SR images were obtained at different incident angles (a) $\theta=62 \mathrm{deg}$ to $67 \mathrm{deg}$. Because of the different penetration depths of the evanescent waves at different incident angles, different parts of the sample were illuminated and excited. As what is shown in the images of the boxed regions (green), the intensity of three parts of the microtubules became weaker as the illumination angle increases, and disappeared at $\theta=65 \mathrm{deg}$. This is consistent with the theory of evanescent wave propagation, that is, when the illumination angle is small, the penetration depth is large, and the signal-to-noise (SNR) is better, when the illumination angle is large, the penetration depth decreases, resulting in the SNR of the sample farther from the cover glass surface decreases until it disappears. Two parts of the microtubules (marked with the purple oval) are more visible at $\theta=65 \mathrm{deg}$, whereas it is not very clear at the $\theta=63 \mathrm{deg}$ and $64 \mathrm{deg}$; this might be due to a decrease in the SNR of the surrounding image, and these two areas themselves are relatively close to the cover glass. This function will be very helpful if someone wants to use this system for axis SR reconstruction to get 3-D SR information. ${ }^{26}$ In addition, when using this system for imaging biological samples, proper adjustment of the illumination angle will be helpful for clear observation of local details or selectively block out some local information that you do not want to see.

Moreover, as shown in Fig. 8, the prototype also has the capability for live cell imaging, which is demonstrated by imaging microtubules in live human bone osteosarcoma epithelial cells (U2OS) labeled with SiR-tubulin (excitation wavelength: $640 \mathrm{~nm} /$ emission wavelength: $660 \mathrm{~nm}$ ). Considering reducing the phototoxicity for long-term exposure, live-cell image series were recorded at 50-ms exposure time per raw image corresponding to a 450-ms SIM frame time, and the nominal illumination intensity for this dataset was $\sim 0.8 \mathrm{~W} \mathrm{~cm}^{-2}$. We can see the swing phenomenon of the microtubules. It is worth noting that the only limitation of the imaging speed in the proposed system is the fluorescent luminescence efficiency and the performance of the camera theoretically, here, means a temporal 

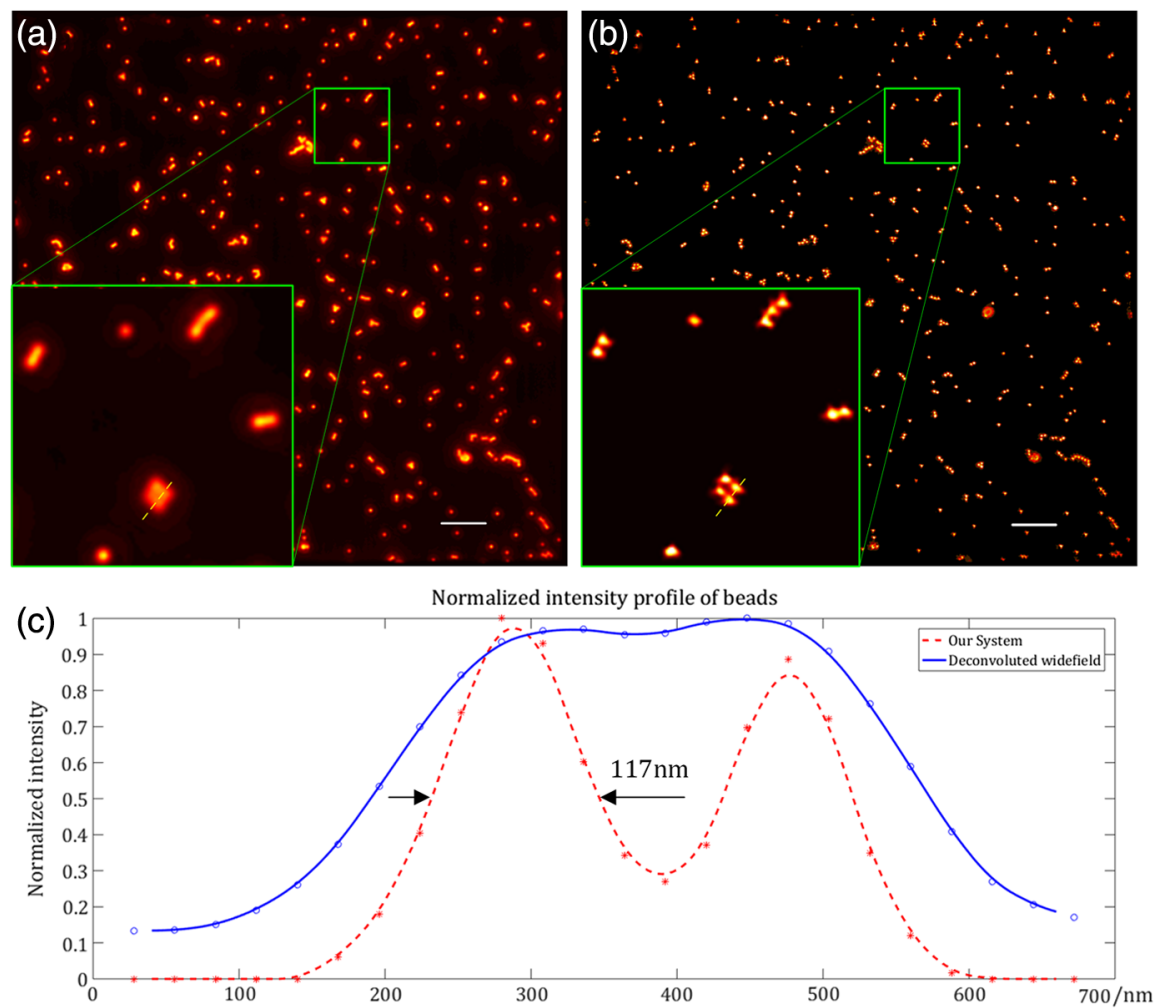

Fig. 4 Results and comparison of $100 \mathrm{~nm}$ fluorescent particles at TIRF-SIM mode. (a) Deconvolved widefield result, (b) reconstructed result of TIRF-SIM using the proposed system, (c) the fluorescence intensity cross profile comparison of the region marked by a yellow line segment in the large inset in (a) and (b). The scale bar is $5 \mu \mathrm{m}$ and the intensity is normalized. The red star dots and blue circle dots are the real cross profile data, and the red line and blue line are the smoothed curves based on these data, representing the intensity of reconstructed image using our SIM system and the deconvolved widefield, respectively.
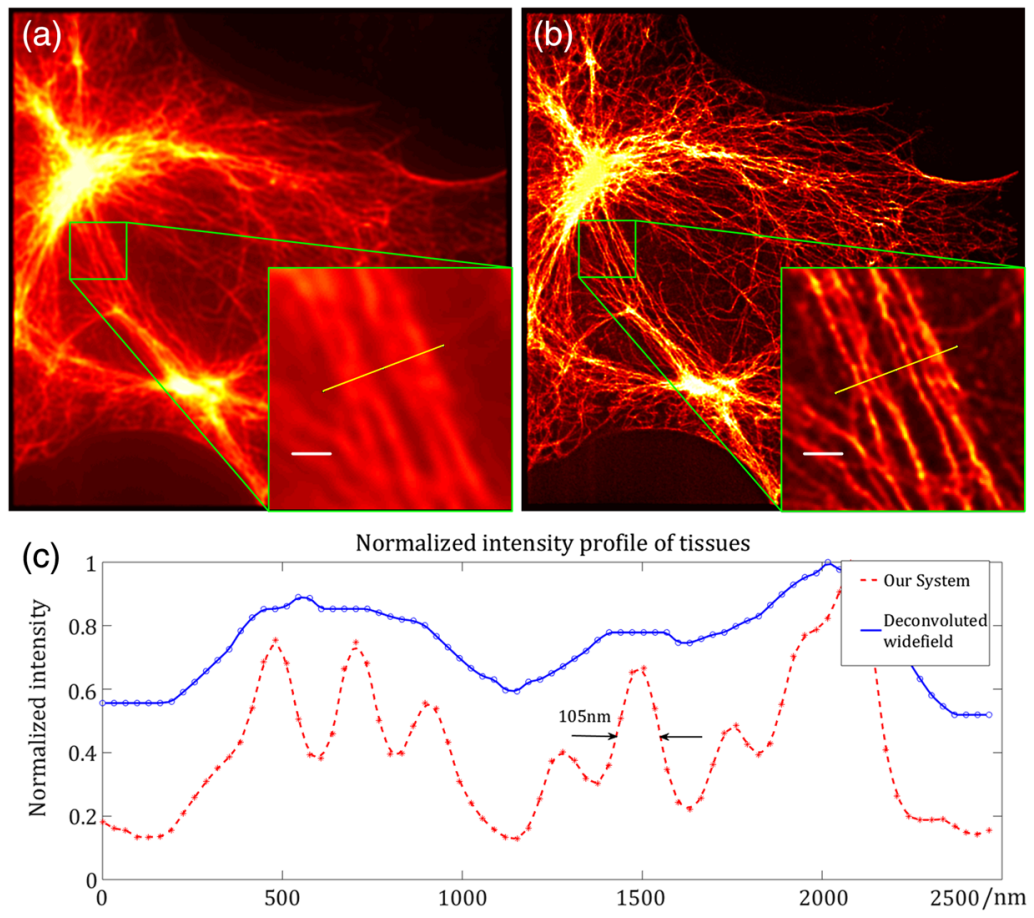

Fig. 5 Results and comparison of microtubules at TIRF-SIM mode. (a) Deconvolved widefield-TIRF result, (b) reconstructed result of TIRF-SIM using the proposed system, and (c) the cross profile of the region marked by a yellow line in (a) and (b). The scale bar is $500 \mathrm{~nm}$ (insets) and the intensity is normalized. The red star dots and blue circle dots are the real cross profile data, and the red line and blue line are the smoothed curves based on these data, representing the intensity of reconstructed image using our SIM system and the deconvolved widefield, respectively. 

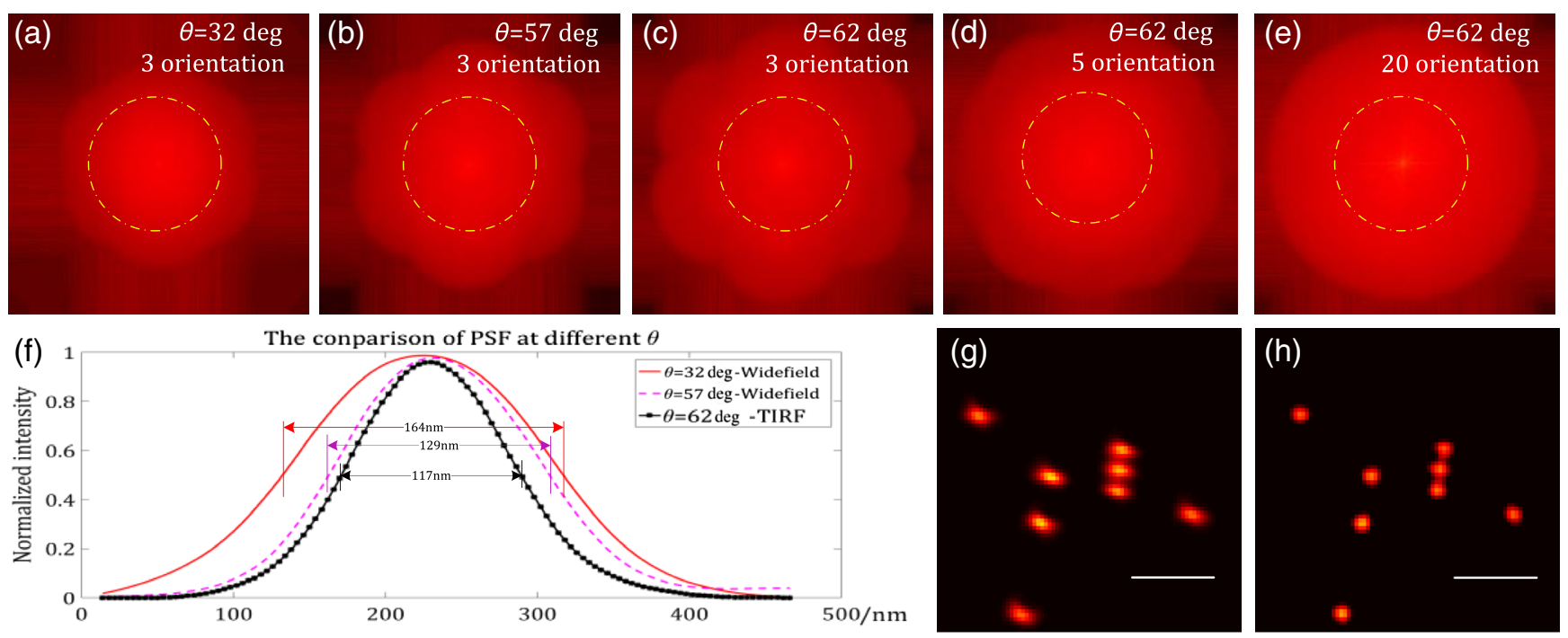

Fig. 6 The frequency components of the reconstructed result and comparison diagram at different angles of incidence and under multiorientation pattern illumination. (a)-(c) The frequency components with the patterns at three orientations with different $\theta$ (32 deg, $57 \mathrm{deg}, 62 \mathrm{deg}$, the corresponding voltages are $0.8,1.45$, and $1.55 \mathrm{~V}$, respectively. These angles are both less than the critical angle and the system works at the widefield-SIM mode); (c)-(e) The spatial frequency components with the patterns at the same $\theta$ and with different orientation $(3,5$, and 20) $(\theta=62$ deg, corresponding voltage is $1.55 \mathrm{~V}$, the system works at the TIRF-SIM mode); (f) the comparison of the FWHMs of the PSFs at different illumination angle; $(\mathrm{g})$ the reconstructed results of beads under the conditions shown in (c); and (h) the reconstructed results of beads under the conditions shown in Fig. 4(e). The scale bar is $500 \mathrm{~nm}$ and the intensity is normalized. The dashed yellow circles in Figs. 4(a)-4(f) represent the maximum spatial frequency supported by the OTF of the system.

resolution of $\sim 10$ SR images per second. In fact, however, for live cell imaging, we should consider the fluorescent brightness, phototoxicity, photobleaching, and the SNR of the image as a whole. There is always a compromise between the imaging speed and the imaging quality.

\section{Conclusion}

We construct and demonstrate a prototype for widefield and TIRF-SIM with scanning galvo mirrors and polarization converter, which can change the illumination directions and angles continuously and conveniently and keep the $s$-polarization interference anytime, offering more flexibility than previous systems. The experimental results proved that a twofold resolution improvement and the live cell imaging capability are achieved by the system. Meanwhile, controlling the pattern period is a requirement for multicolor imaging with different excitation wavelengths and different penetration depth imaging. Furthermore, our prototype also has the capability of
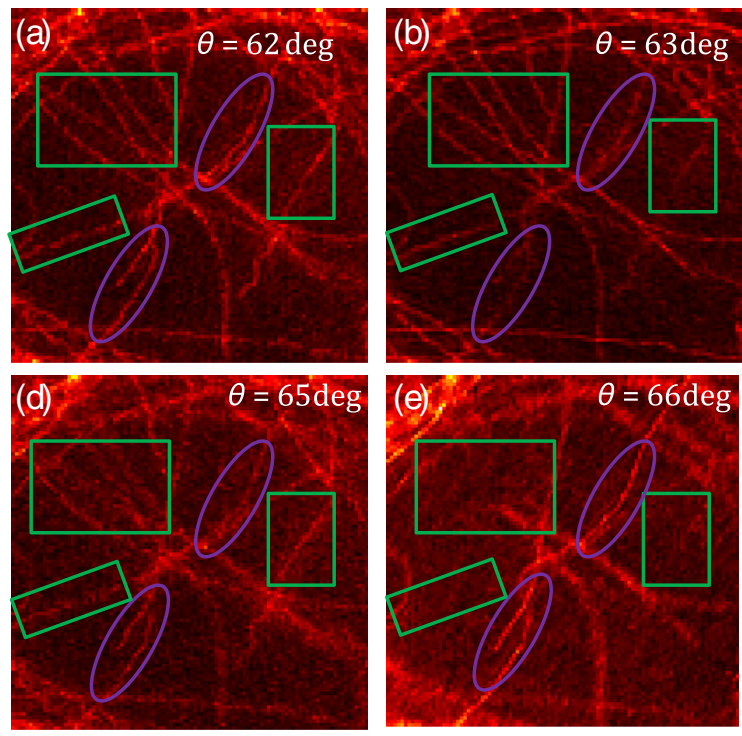
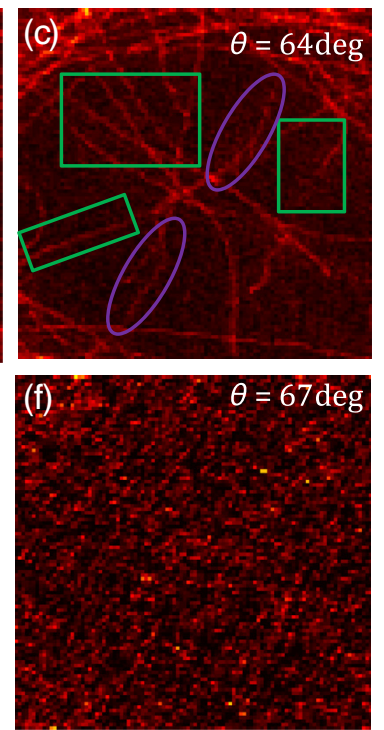

Fig. 7 Optical sectioning capacity of TIRF-SIM. (a)-(f) The reconstructed TIRF-SIM image obtained at $\theta=62 \mathrm{deg}, 63 \mathrm{deg}, 64 \mathrm{deg}, 65 \mathrm{deg}, 66 \mathrm{deg}$, and $67 \mathrm{deg}$, respectively. The scale bar is $5 \mu \mathrm{m}$ and the intensity is normalized. 

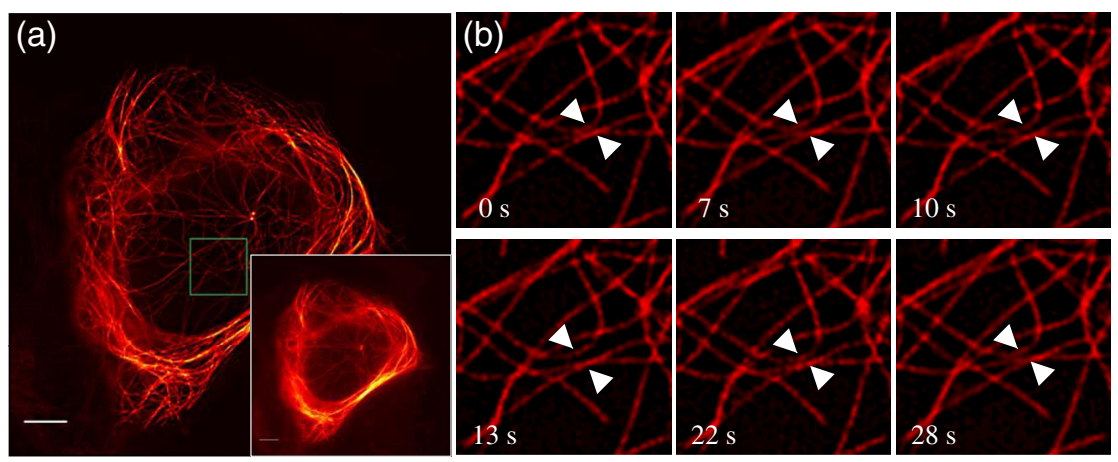

Fig. 8 Live-cell time-lapse imaging of microtubules. (a) TIRF-SIM image of microtubules in live U2OS cells labeled with SiR-tubulin. The right bottom shows the corresponding wide-field image. (b) A timelapse SR image sequence of the ROI marked with green box in (a), which show the changes of lateral positions of microtubules. The scale bar is $5 \mu \mathrm{m}$ and the intensity is normalized.

TIRF-based microscopy, which improves the axial resolution by recording a series of images at different incident angles, or at different wavelengths.

\section{Disclosures}

The authors have no relevant financial interests in this article and no potential conflicts of interest to disclose.

\section{Acknowledgments}

This work was financially sponsored by the National Natural Science Foundation of China (NSFC) (61427818, 61335003, 61505179, and 61735017); the National Basic Research Program of China (973Program) (2015CB352003); the National Key Research and Development Program of China (2017YFC0110303 and 2016YFF0101400); the Natural Science Foundation of Zhejiang province (LR16F050001); and the Fundamental Research Funds for the Central Universities (2017FZA5004).

\section{References}

1. S. W. Hell, "Far-field optical nanoscopy," Science 316(5828), 1153 1158 (2007).

2. S. W. Hell and J. Wichmann, "Breaking the diffraction resolution limit by stimulated emission: stimulated-emission-depletion fluorescence microscopy," Opt. Lett. 19(11), 780-782 (1994).

3. M. G. L. Gustafsson, "Surpassing the lateral resolution limit by a factor of two using structured illumination microscopy," J. Microsc. 198(2), 82-87 (2000)

4. M. G. L. Gustafsson, "Nonlinear structured-illumination microscopy: wide-field fluorescence imaging with theoretically unlimited resolution," Proc. Natl. Acad. Sci. U. S. A. 102(37), 13081-13086 (2005).

5. F. Ströhl and C. F. Kaminski, "Frontiers in structured illumination microscopy," Optica 3(6), 667-677 (2016).

6. M. J. Rust, M. Bates, and X. Zhuang, "Sub-diffraction-limit imaging by stochastic optical reconstruction microscopy (STORM)," Nat. Methods 3(10), 793-796 (2006).

7. B. Huang et al., "Three-dimensional super-resolution imaging by stochastic optical reconstruction microscopy," Science 319(5864), 810-813 (2008)

8. E. Betzig et al., "Imaging intracellular fluorescent proteins at nanometer resolution," Science 313(5793), 1642-1645 (2006).

9. M. Saxena, G. Eluru, and S. S. Gorthi, "Structured illumination microscopy," Adv. Opt. Photonics 7(2), 241-275 (2015).

10. M. G. L. Gustafsson et al., "Three-dimensional resolution doubling in wide-field fluorescence microscopy by structured illumination," Biophys. J. 94(12), 4957-4970 (2008).
11. R. Fiolka, M. Beck, and A. Stemmer, "Structured illumination in total internal reflection fluorescence microscopy using a spatial light modulator," Opt. Lett. 33(14), 1629 (2008).

12. L. J. Young, F. Ströhl, and C. F. Kaminski, "A guide to structured illumination TIRF microscopy at high speed with multiple colors," J. Visualized Exp. 111, 53988 (2016).

13. M. Brunstein et al., "Full-field dual-color 100-nm super-resolution imaging reveals organization and dynamics of mitochondrial and ER networks," Opt. Express 21(22), 26162-26173 (2013).

14. P. Kner et al., "Super-resolution video microscopy of live cells by structured illumination," Nat. Methods 6(5), 339-342 (2009).

15. L. Shao et al., "Super-resolution 3D microscopy of live whole cells using structured illumination," Nat. Methods 8(12), 1044-1046 (2011).

16. D. Li et al., "Extended-resolution structured illumination imaging of endocytic and cytoskeletal dynamics," Science 349(6251), aab3500 (2015).

17. D. Axelrod, "Total internal reflection fluorescence microscopy in cell biology," Traffic 2(11), 764-774 (2001).

18. K. O'Holleran and M. Shaw, "Polarization effects on contrast in structured illumination microscopy," Opt. Lett. 37(22), 4603 (2012).

19. D. Dan et al., "DMD-based LED-illumination super-resolution and optical sectioning microscopy," Sci. Rep. 3, 1116 (2013).

20. M. C. Dos Santos et al., "Topography of cells revealed by variable-angle total internal reflection fluorescence microscopy," Biophys. J. 111(6), 1316-1327 (2016)

21. E. Soubies et al., "A framework for multi-angle TIRF microscope calibration," in IEEE 13th Int. Symp. on Biomedical Imaging (ISBI 2016), pp. 668-671 (2016).

22. K. Wicker et al., "Phase optimisation for structured illumination microscopy," Opt. Express 21(2), 2032-2049 (2013).

23. E. Chung, D. Kim, and P. T. So, "Extended resolution wide-field optical imaging: objective-launched standing-wave total internal reflection fluorescence microscopy," Opt. Lett. 31(7), 945 (2006).

24. J. Demmerle et al., "Strategic and practical guidelines for successful structured illumination microscopy," Nat. Protocol 12(5), 988-1010 (2017).

25. M. Müller et al., "Open-source image reconstruction of super-resolution structured illumination microscopy data in ImageJ," Nat. Commun. 7, 10980 (2016)

26. C. Zheng et al., "Three-dimensional super-resolved live cell imaging through polarized multi-angle TIRF," Opt. Lett. 43(7), 1423-1426 (2018).

Youhua Chen received his BS degree in physics from the Yangzhou University in 2007 and PhD in optical engineering at the Northern University of China in 2013. He is a postdoctor in optical engineering at Zhejiang University and an associate professor at North University of China. He is interested in research work on fluorescence super-resolution microscopy, and mainly takes part in the research work on structure illumination microscopy and its application in biomedicine.

Ruizhi Cao is currently an undergraduate student of College of Optical Science and Engineering, Zhejiang University. He mainly 
focuses on developing new fluorescence imaging methods and image reconstruction algorithms at present.

Wenjie Liu is currently a doctoral student at Zhejiang University, Hangzhou, China, under the supervision of Dr. Cuifang Kuang. His research interests are the development of novel super-resolution imaging techniques including total internal reflection microscopy, structured illumination microscopy and 3-D fluorescence emission difference microscopy.

Dazhao Zhu received his master's degree in Optoelectronic Engineering Department of Changchun University of Science and Technology. Currently, he is a doctoral student at Zhejiang University, Hangzhou, China, under the supervision of Dr. Cuifang Kuang. His current research focuses on super-resolution imaging based on fluorescence emission difference microscopy.

Zhiming Zhang is currently a doctoral student at Zhejiang University, Hangzhou, China, under the supervision of Dr. Cuifang Kuang. His current research interests are the development of super-resolution imaging techniques including optical fluctuation imaging, array scan imaging, and structured illumination microscopy.

Cuifang Kuang received his PhD from Beijing Jiaotong University in 2007. Currently, he is a professor at Zhejiang University, Hangzhou, China. He recently focuses on optical super-resolution imaging techniques. He authored more than 80 journal publications, including physical reviews letter, light: science \& applications, optica, scientific reports, optics letter and optics express, and so on, 60 patents, 2 book chapters.

Xu Liu is a professor at Zhejiang University, Hangzhou, China. He focuses on optical films coatings and coating techniques; optical meta-surface, micro- and nanostructured devices; real 3-D display, projection display, AR/VR display, and 3-D interaction techniques; optical super-resolution imaging, computation imaging, bioimaging, and detection; and free-form optics for illumination and for imaging. $\mathrm{He}$ is coauthor of over 300 publications. 